\title{
NOUVELle
}

\section{Le nez au chevet du cerveau}

Emmanuel Nivet, Arnaud Devèze, Stéphane D. Girard, François S. Roman, François Féron

> La cavité nasale abrite la muqueuse olfactive, le seul tissu nerveux qui soit à la fois accessible chez tout individu et en contact direct avec l'environnement. Grâce à des cellules souches nombreuses et fortement prolifératives, ce tissu se régénère en permanence. Plusieurs travaux, conduits dans des modèles animaux d'amnésie, de paraplégie, de surdité et de la maladie de Parkinson, indiquent que la médecine régénérative ne manquera pas de flair si elle fait de ce nouveau type de cellules souches adultes l'un de ses candidats favoris pour des essais cliniques de transplantation autologue.

La thérapie cellulaire représente un espoir grandissant dans la course de l'homme contre le temps et les éléments. Dans la lignée du concept proposé par Hippocrate puis Paracelse ${ }^{1}$ (simila similibus curatur, le semblable guérit le semblable), les premières greffes, destinées à réparer un organe lésé ou pathologique, utilisaient des cellules issues du même organe mais en provenance d'un animal ou un individu sain. Puis, vers la fin $d u x x^{e}$ siècle, est apparue la notion de plasticité cellulaire. Au lieu de faire appel à une cellule spécialisée, peu plastique, cliniciens et chercheurs spécialistes de la médecine régénératrice se sont intéressés aux cellules souches. Ces dernières présentent une grande versatilité phénotypique en réponse à des signaux extérieurs, et sont douées d'une adap-

1 Philippus, Aureolus, Theophrastus Bombast von Hohenheim, dit Paracelse (1493-1541). Il s'intitulait Prince des deux médecines - celle du corps et celle de l'âme, et avait pour particularité de porter toujours une longue épée qu'il tenait, dit-on, d'un bourreau et dont le pommeau aurait renfermé la Pierre des Sages. tabilité environnementale. Les cellules souches à fort potentiel thérapeutique peuvent être regroupées en deux grandes catégories : (1) pluripotentes, généralement d'origine embryonnaire ou issues de reprogrammation cellulaire ; (2) multipotentes, isolées à partir de tissus adultes le plus souvent. Si les cellules souches pluripotentes, ayant la capacité de générer pratiquement tous les types cellulaires, offrent d'énormes espoirs, les problèmes éthiques et techniques qui y sont associés ralentissent leur utilisation dans le domaine clinique [11]. À l'inverse, bien que plus limitées dans leur potentiel de différenciation, les cellules souches adultes représentent une alternative de choix et peuvent être isolées à partir de nombreuses sources tissulaires [12].

\section{Cellules souches de la muqueuse}

olfactive : un fort potentiel

\section{thérapeutique}

Chaque tissu, soumis à un renouvellement cellulaire au cours de la vie adulte, abrite généralement un pool de cellules souches permettant le maintien de ce processus. Parmi celles-ci, les cellules souches neurales sont les cellules mères des tissus neuraux [13]. Dans ce contexte, les cellules souches neurales apparaissent comme un outil de choix pour des transplantations destinées à compenser les pertes cellulaires pathologiques ou traumatiques du système nerveux central.
Néanmoins, leur localisation dans des couches profondes du cerveau rend leur utilisation difficile. Afin de contourner cet obstacle, nous avons recherché un nouveau type de cellule candidate qui soit à la fois facilement accessible et prédisposée à se différencier en cellules neurales. Partant du principe que la muqueuse olfactive humaine est un tissu nerveux périphérique, siège d'une neurogenèse permanente, nous avons émis I'hypothèse qu'un type cellulaire résident pourrait être d'intérêt pour la réparation de réseaux neuronaux et gliaux.

En 2005, nous avons identifié pour la première fois l'existence d'un nouveau type de cellules souches, localisées dans la zone sous épithéliale de la muqueuse olfactive nommée lamina propria et possédant des propriétés de multipotentialité [1]. Ces cellules présentent la remarquable capacité de se différencier en cellules neurales (neurones, astrocytes, oligodendrocytes) mais également en d'autres types cellulaires (cellules 
sanguines, musculaires ou cardiaques) lorsqu'elles sont placées, in vitro et/ ou in vivo, dans un environnement propice. Ces cellules présentent un spectre de différenciation bien plus large que toutes les autres cellules souches adultes décrites à ce jour et font montre d'une grande plasticité/adaptabilité en réponse aux signaux externes (développementaux ou adultes). Plus récemment, nous avons caractérisé ces cellules qui présentent de nombreuses similitudes géniques et protéiques avec les cellules souches mésenchymateuses de la moelle osseuse $[2,12]$. De manière très intéressante, les cellules souches mésenchymateuses sont aujourd'hui utilisées dans de nombreux essais cliniques de transplantation cellulaire $(\rightarrow)$ [14].

Ce nouveau type de cellule souche, qui

$(\rightarrow)$ Voir à ce sujet le numéro thématique de $\mathrm{m} / \mathrm{s}$ Cellules souches mésenchymateuses, $n^{\circ} 3$, vol. 27 , mars 2011

combine certaines caractéristiques des cellules souches mésenchymateuses et des cellules souches neurales, nous est donc apparu comme un parfait candidat pour des expériences de thérapie neurale. En 2008, à l'aide d'un modèle animal de la maladie de Parkinson, nous avons apporté la première démonstration que des cellules souches de la muqueuse olfactive humaine étaient capables de rétablir des fonctions perdues lorsqu'elles sont transplantées dans les zones cérébrales affectées par une neurodégénérescence [3]. De plus, l'équipe de Roisen aux États-Unis a démontré que la transplantation de cellules souches olfactives nasales humaines améliorait significativement la locomotion de rats devenus paraplégiques après une section de la moelle épinière [4]. Parallèlement, la capacité de différenciation de ces mêmes cellules humaines en chondrocytes, après transplantation dans un modèle rat de lésion des disques intervertébraux [5], ou en ostéocytes après insertion chez des souris nude, a été mise en évidence [2]. Enfin, une troisième équipe a apporté la preuve que: (1) les cellules
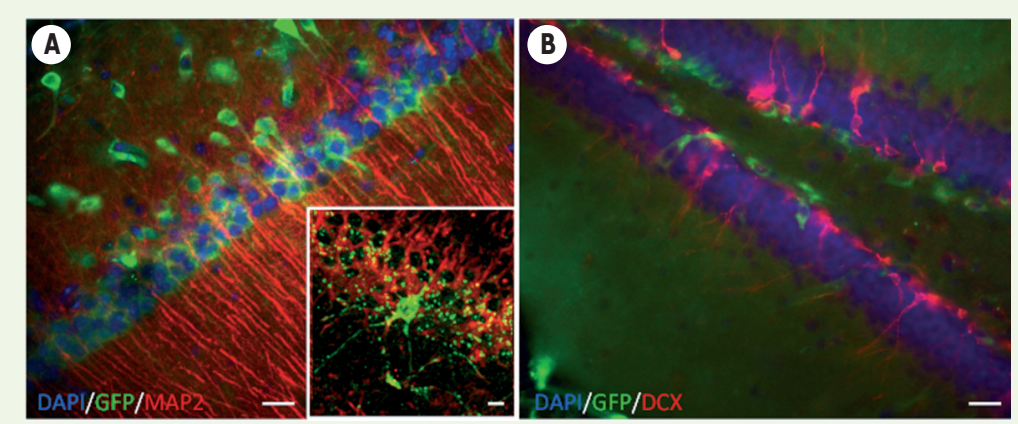

Figure 1. Transplantation intracérébrale ou intraventriculaire de cellules souches de la muqueuse olfactive. Cinq semaines après transplantation, des cellules souches humaines de la muqueuse olfactive (GFP+/vertes) sont retrouvées dans les couches cellulaires hippocampiques des champs amoniens $(\boldsymbol{A})$ et du gyrus dentelé $(\boldsymbol{B})$. Les cellules humaines, après avoir migré, se sont intégrées aux réseaux neuronaux préexistants et possèdent majoritairement des caractéristiques phénotypiques [8] et morphologiques (insert en $A$ ) de neurones. De plus, les cellules humaines stimulent la formation de nouveaux neurones endogènes exprimant la doublecortine $(D C X+/$ rouge $)(B)$. (barres d'échelles : $20 \mu \mathrm{m}$ ).

souches olfactives de souris pouvaient se différencier in vitro en cellules ciliées cochléaires [6], et (2) les cellules souches olfactives humaines, transplantées chez des souris présentant des pertes de cellules ciliées, aidaient à préserver la fonction auditive [7].

\section{Cellules souches olfactives:} restauration des fonctions mnésiques Encore plus récemment, nous avons évalué l'effet d'une transplantation de cellules souches de la muqueuse olfactive humaine dans un modèle consistant à induire la mort d'un grand nombre de neurones hippocampiques chez la souris [8]. Les hippocampes jouent un rôle central dans le système limbique, participant notamment aux processus d'apprentissage et de mémorisation. Cette structure constitue le support fonctionnel à la mise en place de nouveaux souvenirs et toute atteinte, traumatique ou pathologique, induisant une mort neuronale excessive (ischémie/hypoxie) et/ou un dysfonctionnement (la maladie d'Alzheimer), engendre un syndrome amnésique. Nous avons montré que, contrairement à leurs congénères non lésées, les souris chez lesquelles on a créé une lésion au niveau des couches cellulaires hippocampiques étaient dans l'incapacité d'apprendre et mémoriser les tâches auxquelles elles étaient soumises. Elles ne parvenaient pas à faire d'association entre une odeur et l'attribution d'une récompense (test du labyrinthe olfactif circulaire). De la même manière, elles étaient dans l'impossibilité de mémoriser l'emplacement d'une plate-forme submergée et invisible lorsqu'elles étaient placées dans une enceinte circulaire remplie d'eau et entourée d'indices visuels (tâche de la piscine de Morris). Notre étude a montré que les atteintes fonctionnelles consécutives à la perte de neurones hippocampiques pouvaient être compensées par des cellules souches humaines de la muqueuse olfactive, transplantées soit directement dans la structure lésée, soit dans le liquide céphalo-rachidien. De manière remarquable, nous avons montré que ces cellules pouvaient survivre en grand nombre (20\%) quatre semaines après la greffe, mais également migrer vers les zones lésées pour s'y différencier en cellules neuronales (Figure 1). Parallèlement, nous avons observé que les cellules exogènes stimulaient la production endogène de nouveaux neurones à partir des cellules souches du gyrus dentelé (Figure I). Enfin, les analyses électrophysiologi- 


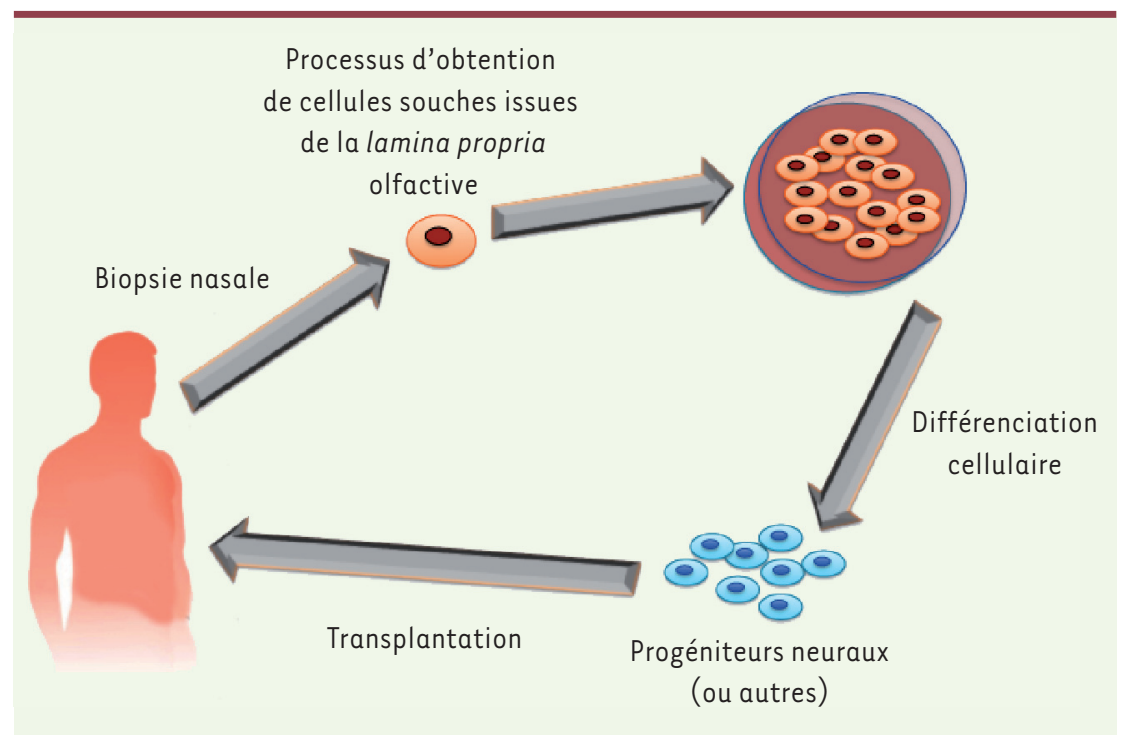

Figure 2. Différentes étapes pour une future utilisation de cellules souches de la muqueuse olfactive autologues en médecine régénérative.

ques des souris transplantées ont révélé un rétablissement partiel de l'activité électrique hippocampique (transmission cellulaire et potentialisation à long terme), à la base de la récupération des fonctions mnésiques observée.

Un bel avenir...

Les données concernant les cellules souches de la muqueuse olfactive convergent et plaident en faveur de leur utilisation dans un cadre thérapeutique. Leur facilité d'accès chez l'homme [9] est un avantage considérable qui permet d'envisager des transplantations autologues, évitant ainsi tout risque de rejet ou d'effets secondaires liés à un traitement immunosuppresseur. La démonstration dans ces différentes études indépendantes du rôle neuroprotecteur et neurostimulateur de cet outil cellulaire, suggère qu'il pourrait bénéficier à toutes les pathologies associées à des pertes de cellules neuronales (Figure 2). De plus, ces cellules devraient pouvoir outrepasser certains obstacles comme la contreindication de transplantation autologue dans le cadre de neuropathologies qui, dans certains cas, peuvent être d'origine génétique (Parkinson, Huntington) et donc engendrer un risque de rechute. En effet, dans une étude de correction génique extrêmement originale nous avons récemment apporté la preuve de principe que ces cellules peuvent être corrigées en amont d'une transplantation [10]. Par ailleurs, lorsqu'elles sont cultivées dans des conditions favorables, ces cellules ont un fort pouvoir proliférant et il est possible de disposer d'un grand nombre de cellules dans un délai relativement court. Notons qu'à ce jour, nous n'avons jamais observé la formation de tumeur après transplantation de ces cellules. Enfin, la capacité d'écotropisme (homing en anglais) de ces cellules en réponse à des signaux postlésionnels en fait un outil de choix pour des greffes peu invasives, notamment par voie intraveineuse. De manière générale, si la médecine de demain s'inscrit toujours dans la recherche de produits cellulaires à fort potentiel thérapeutique, il fait peu de doutes que les cellules souches de la muqueuse olfactive humaine doivent être considérées comme d'excellentes candidates. $\diamond$

\section{Nose at the bedside of the brain}

\section{CONFLIT D'INTÉRÊTS}

Les auteurs déclarent n'avoir aucun conflit d'intérêts concernant les données publiées dans cet article.

\section{RÉFÉRENCES}

1. Murrell W, Feron F, Wetzig A, et al. Multipotent stem cells from adult olfactory mucosa. Dev Dyn 2005; $233: 496-515$.

2. Delorme B, Nivet $\varepsilon$, Gaillard J, et al. The human nose harbors a niche of olfactory ectomesenchymal stem cells displaying neurogenic and osteogenic properties. Stem Cells Dev 2010 ; 19 : 853-66.

3. Murrell W, Wetzig A, Donnellan M, et al. Olfactory mucosa is a potential source for autologous stem cell therapy for Parkinson's disease. Stem Cells 2008 ; 26 : 2183-92.

4. Xiao M, Klueber KM, Guo Z, et al. Human adult olfactory neural progenitors promote axotomized rubrospinal tract axonal reinnervation and locomotor recovery. Neurobiol Dis $2007 ; 26: 363-74$.

5. Murrell W, Sanford E, Anderberg L, et al. Olfactory stem cells can be induced to express chondrogenic phenotype in a rat intervertebral disc injury model. Spine J $2009 ; 9: 585-94$.

6. Doyle KL, Kazda A, Hort Y, et al. Differentiation of adult mouse olfactory precursor cells into hair cells in vitro. Stem Cells $2007 ; 25: 621-7$.

7. Pandit SR, Sullivan JM, Egger V, et al. Functional effects of adult human olfactory stem cells on earlyonset sensorineural hearing loss. Stem Cells 2011 ; $29: 670-7$.

8. Nivet $\varepsilon$, Vignes M, Girard SD, et al. Engraftment of human nasal olfactory stem cells restores neuroplasticity in mice with hippocampal lesions. J Clin Invest 2011 ; 121 : 2808-20.

9. Girard SD, Devèze $A$, Nivet $\varepsilon$, et al. Isolating nasal olfactory stem cells from rodents or humans. J Vis Exp 2011. http://www.jove.com/details.php?id=2762

10. Liu GH, Suzuki K, Qu J, et al. Targeted gene correction of laminopathy-associated LMNA mutations in patient-specific iPSCs. Cell Stem Cell $2011 ; 8$ : 688-94.

11. Kieffer $\varepsilon$, Kuntz $S$, Viville $S$. Tour d'horizon des lignées de cellules souches pluripotentes. Med Sci (Paris) $2010 ; 26: 848-54$.

12. Charbord P, Casteilla L. La biologie des cellules souchesmésenchymateuses d'origine humaine. Med Sci (Paris) 2011 ; $27: 261-7$.

13. De Chevigny $A$, Lledo PM. La neurogenèse bulbaire et son impact neurologique. Med Sci (Paris) 2006 ; 22 : 607-13.

14. Sensebé $L$ (coordinateur). Cellules souches mésenchymateuses ( $\mathrm{n}^{\circ}$ thématique). Med Sci (Paris) $2011 ; 27: 261-307$.
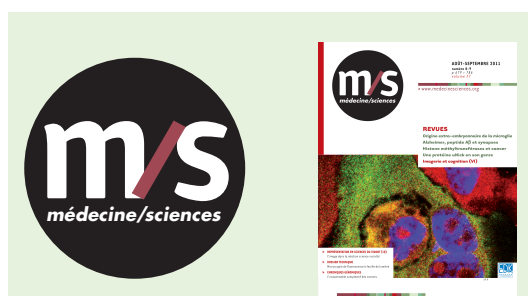

Abonnez-vous

à médecine/sciences

Bulletin d'abonnement page 952 dans ce numéro de $\mathrm{m} / \mathrm{s}$ 